\title{
Colorectal liver metastatic disease: efficacy of irreversible electroporation-a single-arm phase II clinical trial (COLDFIRE-2 trial)
}

Hester J. Scheffer ${ }^{1 *}$, Laurien G P H Vroomen ${ }^{1}$, Karin Nielsen², Aukje A J M van Tilborg ${ }^{1}$, Emile F I Comans ${ }^{1}$, Cornelis van Kuijk', Bram B. van der Meijs', Janneke van den Bergh', Petrousjka M P van den Tol ${ }^{2}$ and Martijn R. Meijerink ${ }^{1}$

\begin{abstract}
Background: Irreversible electroporation (IRE) is a novel image-guided tumor ablation technique that has shown promise for the ablation of lesions in proximity to vital structures such as blood vessels and bile ducts. The primary aim of the COLDFIRE-2 trial is to investigate the efficacy of IRE for unresectable, centrally located colorectal liver metastases (CRLM). Secondary outcomes are safety, technical success, and the accuracy of contrast-enhanced (ce)CT and ${ }^{18}$ F-FDG PET-CT in the detection of local tumor progression (LTP).

Methods/design: In this single-arm, multicenter phase II clinical trial, twenty-nine patients with ${ }^{18} \mathrm{~F}$-FDG PET-avid $C R L M \leq 3,5 \mathrm{~cm}$ will be prospectively included to undergo IRE of the respective lesion. All lesions must be unresectable and unsuitable for thermal ablation due to vicinity of vital structures. Technical success is based on ceMRI one day post-IRE. All complications related to the IRE procedure are registered. Follow-up consists of ${ }^{18}$ F-FDG PET-CT and 4-phase liver CT at 3-monthly intervals during the first year of follow-up. Treatment efficacy is defined as the percentage of tumors successfully eradicated 12 months after the initial IRE procedure based on clinical follow-up using both imaging modalities, tumor marker and (if available) histopathology. To determine the accuracy of ${ }^{18} \mathrm{~F}-\mathrm{FDG}$ PET-CT and ceCT, both imaging modalities will be individually scored by two reviewers that are blinded for the final oncologic outcome.

Discussion: To date, patients with a central CRLM unsuitable for resection or thermal ablation have no curative treatment option and are given palliative chemotherapy. For these patients, IRE may prove a life-saving treatment option. The results of the proposed trial may represent an important step towards the implementation of IRE for central liver tumors in the clinical setting.
\end{abstract}

Trial registration: Trial registration number: NCT02082782.

Keywords: Irreversible electroporation (IRE), Liver neoplasms/secondary, Local, FDG-PET, PET-CT, Tumor ablation, Neoplasm recurrence, Colorectal liver metastases

\section{Background}

Colorectal cancer causes each 10th death due to cancer in Western countries. About $33 \%$ of all patients with colon cancer have liver-only metastatic disease [1]. For these patients, surgical resection is the gold standard, with 5-year survival rates up to $60 \%[2,3]$. However,

\footnotetext{
* Correspondence: hj.scheffer@vumc.nl

'Department of Radiology and Nuclear Medicine, VU University Medical Center, de Boelelaan 1117, 1081 HV Amsterdam, The Netherlands Full list of author information is available at the end of the article
}

despite improvements of surgical techniques and current neoadjuvant chemotherapies, only 5-20\% of patients with colorectal liver metastases (CRLM) can benefit from surgery due to number, localization, or distribution of tumors [4].

In light of the limitations of surgical resection for many hepatic tumors, a number of ablative technologies for liver-directed therapy have developed during the last 20 years [5]. Of these techniques, thermal ablation using radiofrequency ablation (RFA) and microwave ablation

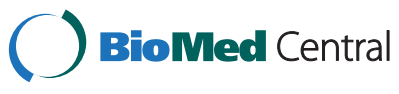

(c) 2015 Scheffer et al. Open Access This article is distributed under the terms of the Creative Commons Attribution 4.0 International License (http://creativecommons.org/licenses/by/4.0/), which permits unrestricted use, distribution, and reproduction in any medium, provided you give appropriate credit to the original author(s) and the source, provide a link to the Creative Commons license, and indicate if changes were made. The Creative Commons Public Domain Dedication waiver (http://creativecommons.org/publicdomain/zero/1.0/) applies to the data made available in this article, unless otherwise stated. 
(MWA) are most frequently used. A recent review showed a local recurrence rate of $2.8-29.7 \%$ of RFablated liver lesions at 12-49 months follow-up, and 2.7-12.5\% of MW-ablated lesions at 5-19 months follow-up, with a 5-year survival rate around $30 \%$ for both techniques [6]. Although thermal ablation has dramatically improved survival rates for patients with CRLM, factors like size and location limit its use and effectiveness. Efficacy of RFA rapidly decreases for lesions $>3 \mathrm{~cm}$ [7]. Also, the rate of complete tumor necrosis falls below $50 \%$ when vessels larger than $3 \mathrm{~mm}$ abut the tumor as a consequence of the heat-sink effect [8]. Ablation of lesions close to vital structures like major bile ducts and vessels is associated with a substantial risk of complications due to thermal damage.

Irreversible electroporation (IRE) is a novel ablation modality that may overcome some of the limitations of current thermal ablation therapies. It is based on a pulsating current created between multiple needle electrodes placed around the target lesion which alters the existing cellular transmembrane potential. If the duration of the applied electrical pulses is below the charging time of the outer cell membrane, an interaction of the electric field with subcellular structures occurs [9]. This interaction results in permanent permeabilization of the cell membrane, which disrupts cellular homeostasis and ultimately leads to cell death. The irreversibly damaged cells are removed by the immune system [10]. Two main factors stimulate research into IRE as an ablation modality. Since the mechanism of cell death is predominantly nonthermal, connective tissue structure is preserved, so there is no damage to associated blood vessels and bile ducts [11-13]. For the same reason, treatment efficacy is not impeded by heat-sink [14]. This allows for treatment of liver tumors deemed unresectable or ineligible for other focal ablation techniques due to localization near these structures [15].

The capability of IRE to destroy CRLM in humans was recently demonstrated in the COLDFIRE-1 ablate-andresect trial [16]. In this trial resectable CRLM were treated with IRE, followed by resection one hour later. The investigators demonstrated cell death of the ablated tumors within one hour after IRE, with intact larger vascular and ductal structures within the ablation zone. The first studies investigating hepatic IRE on clinical indication also yield promising results. A systematic review found an overall complication rate of $16 \%$ for hepatic IRE, similar to RFA [17]. However, the tumors treated with IRE were all located near thermally sensitive structures, as opposed to the thermally ablated lesions. Complete tumor eradication was achieved in $67-100 \%$, and this percentage was even higher for tumors $<3 \mathrm{~cm}$ [17]. However, since most studies are retrospective with short-term follow-up using heterogeneous study populations and design, the value of the current evidence remains limited. Also, the optimal imaging modality for follow-up after IRE needs to be analyzed.

The primary aim of the COLDFIRE-2 trial is to investigate the efficacy of IRE for CRLM that are unsuitable for resection and thermal ablation due to the vicinity of vulnerable structures such as bile ducts, vessels and bowel. Other outcomes are safety of IRE, and the accuracy of contrast-enhanced computed tomography (ceCT) and ${ }^{18} \mathrm{~F}$-fluorodeoxyglucose (FDG) positron emission tomography (PET) CT in the detection of local tumor progression (LTP).

\section{Methods/design \\ Design}

The COLDFIRE-2 trial is a phase II, multicenter, prospective single-arm trial that is organized by the VU University Medical Center in Amsterdam, the Netherlands. Patients will be recruited at three academic hospitals in the Netherlands (VU University Medical Center, Amsterdam; Academic Medical Center, Amsterdam; Leiden University Medical Center, Leiden). The study protocol has been approved by the Medical Ethical Review Board of the VU University Medical Center. The trial is investigatorsponsored, independent of industry and is registered at clinicaltrials.gov under number NCT02082782. The trial will be conducted in accordance with the Declaration of Helsinki. The inclusion and exclusion criteria for the COLDFIRE- 2 trial are summarized in Table 1.

\section{Eligibility criteria}

All patients are treated with curative intent and must have received previous chemotherapy for CRLM at some stage in their disease. Prior to inclusion, all patients will be discussed in a multidisciplinary liver tumor board consisting of a hepatogastroenterologist, hepatobiliary surgeon, medical oncologist, radiation oncologist, abdominal and interventional radiologist. Decision on treatment will

Table $1 \mathrm{ln}$ - and exclusion criteria

\begin{tabular}{ll}
\hline Inclusion criteria & Exclusion criteria \\
\hline $\begin{array}{l}\text { Histologic proof of primary } \\
\text { colorectal tumor }\end{array}$ & $\begin{array}{l}\text { Ventricular cardiac } \\
\text { arrhythmias } \\
\text { Radiologic proof of colorectal liver } \\
\text { metastasis, unsuitable for resection } \\
\text { and thermal ablation due to vicinity } \\
\text { to vascular or ductal structures }\end{array}$ \\
$\begin{array}{l}\text { Congestive heart failure, } \\
\text { NYHA Class } \geq 3\end{array}$ \\
and visible on ceCT & \\
Lesion size $\leq 3.5 \mathrm{~cm}$ & Active coronary artery \\
Adequate bone marrow, liver & disease \\
and renal function & History of epilepsy \\
ASA-classification $0-3$ & Any implanted stimulation \\
& device \\
\hline
\end{tabular}


be at their discretion. Patients who present with more than one metastasis can only be included if treatment with curative intent is still realistic and if all lesions can be treated during the same session or within six weeks after the IRE procedure. Thermal ablation or resection of concomitant lesions during the same session is therefore allowed and limited extrahepatic disease, defined as $\leq 5$ nodules in the lung and/or one other metastatic site which is amenable to future definitive treatment, is not contraindicated [18]. All participants from all participating centers will provide written informed consent.

\section{IRE procedure}

Depending on concurrent treatment of other lesions (surgical resection or thermal ablation), patients will either be treated during an open procedure using intraoperative ultrasound (IOUS), or percutaneously using CT. Patients undergoing laparotomy will receive a thoracic epidural before surgery. For percutaneous procedures, to allow repeated and real-time visualization of both the tumor and the adjacent vessels, a catheter is placed within the common hepatic artery approaching from the right femoral artery. Technical details of this procedure have been previously described by Van Tilborg et al. [19]. All procedures will be performed under general anesthesia, induced with propofol, sufentanil, and rocuronium and maintained with propofol and remifentanil. First, the exact three-dimensional measurements of the target lesion are defined using IOUS or aortic catheter based CT. The planned electrode configuration must result in an expected geometry of the ablation zone that fully covers the tumor and a tumor-free margin of at least $5 \mathrm{~mm}$ in all directions [20]. Depending on the size, 2-6 needle electrodes (NanoKnife, AngioDynamics, Latham, NY) with an active working length of $20 \mathrm{~mm}$ are positioned in the outer border or just adjacent to the tumor under IOUS or CT guidance, aiming at an inter-electrode distance of $20 \mathrm{~mm}( \pm 2 \mathrm{~mm})$. All needles are placed parallel to each other to promote homogeneous energy delivery. After confirmation of correct distances with IOUS or with unenhanced CT using multiplanar reformatting, ten testpulses of $1500 \mathrm{~V} / \mathrm{cm}$ and $90 \mu \mathrm{s}$ are delivered via each electrode pair, after which the delivered current is verified. The target current lies between $20-50 \mathrm{~A}$ and voltage settings are manually adjusted in case of over- or undercurrent. Subsequently, three cycles of 30 pulses are administered to reach a total of 100 pulses per electrode pair. If more than 6 electrodes are needed for larger tumors, electrodes are repositioned to perform overlapping ablations. Similarly, for tumors with a depth larger than $20 \mathrm{~mm}$, after ablation of the deepest part of the tumor a $1.5 \mathrm{~cm}$ pullback of the electrodes is performed to treat the superficial part. Immediately after IRE, a control aortic catheter CT or IOUS is made to evaluate technical success and to exclude early complications. The next day, ceMRI is performed to verify patency of vascular and ductal structures, as well as technical success. In case of incomplete ablation on ceMRI, the suspected residual tumor will be retreated in the following weeks. A flow diagram of the trial is shown in Fig. 1.

\section{Imaging}

Four-phase liver CT and ${ }^{18}$ F-FDG PET-CT will be performed before IRE, and 3, 6, 9 and 12 months after IRE. Imaging is conducted according to EANM guidelines with the same ${ }^{18} \mathrm{~F}$-FDG PET-CT in each participating center (Philips Gemini TF PET-CT system, Philips Medical Systems, Cleveland, Ohio, USA) [21]. Whole body ${ }^{18} \mathrm{~F}-$ FDG PET (skull base to mid-thigh) starts $60 \mathrm{~min}$ after FDG injection, followed by a low-dose CT for attenuation correction and anatomical co-localization of ${ }^{18} \mathrm{~F}$-FDG PET-findings. Next, a diagnostic 4-phase CT of the liver is performed. Scanning parameters are shown in Table 2.

Treatment response is primarily based on a per-lesion analysis. The standard of reference is defined by a combination of 4-phase liver CT and ${ }^{18} \mathrm{~F}$-FDG PET-CT, and, if available, histologically proven malignancy. Pathologically elevated carcinoembryonic antigen (CEA) is used when appropriate (e.g. if no other metastases are present).

Treatment response on 4-phase liver CT is assessed using the Response Evaluation Criteria In Solid Tumors (RECIST) [22]. The Positron Emission tomography Response Criteria In Solid Tumors (PERCIST) criteria are used for ${ }^{18} \mathrm{~F}$-FDG PET-CT [23]. On 4-phase liver CT, LTP is defined as a growing ( $>20 \%$; longest diameter; axial plane) hypodense lesion within $1 \mathrm{~cm}$ of the ablation zone. On ${ }^{18}$ F-FDG PET-CT, focally increased FDG uptake within $1 \mathrm{~cm}$ of the ablation region is considered an LTP [24]. If LTP is suspected on both modalities during follow-up, the patient will be evaluated for re-treatment in our multidisciplinary liver tumor board. To establish the value of PET-CT and ceCT for the prediction of LTP the suspected area will be biopsied in the same session prior to re-treatment, although the suspicious lesion will be re-ablated regardless. In case of discrepancy between the 4-phase liver CT and ${ }^{18}$ F-FDG PET-CT findings, a liver MRI will be performed as problem solver. If MRI is also inconclusive, multidisciplinary deliberation will decide upon either repeat imaging 3 months later, or biopsy of the suspected recurrence. If chemotherapy is indicated during followup (e.g. due to new metastatic disease), the patient will remain in follow-up. The trial will end twelve months after the last IRE procedure. If no LTP or new metastatic disease has been diagnosed at this moment, regular follow-up will be resumed consisting of 6-monthly ${ }^{18}$ F-FDG PET-CT and 4-phase liver CT. 


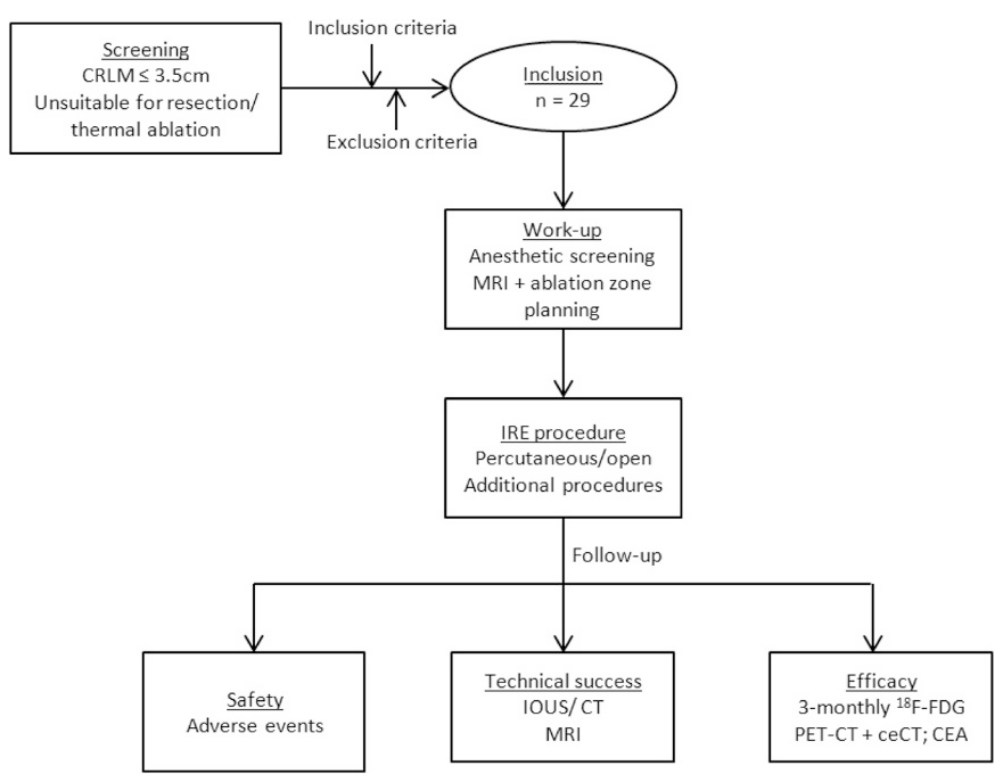

Fig. 1 Flow diagram of study procedure

\section{Data collection and handling}

The study coordinators (HS and LV) will collect the data. All data will be handled confidentially and anonymously. A subject identification code is used to link the data to the subject. The study coordinators safeguard the key to the identification code. The handling of personal data will comply with the Dutch Personal Data Protection Act.

To investigate the accuracy of ${ }^{18} \mathrm{~F}$-FDG PET-CT and 4-phase liver CT in the diagnosis of LTP after IRE, twelve months after the last IRE treatment blinded data sets from all centers will be reviewed separately and independently by two designated radiologists (BvdM and $\mathrm{JvdB})$ and two nuclear medicine physicians $(\mathrm{OH}$ and EC) in a consensus reading. Each lesion will be scored on a separate form on a one to five scale (Table 3) [25]. The reviewers will be blinded to the results of the other imaging modality and to the final oncologic outcome as determined by the standard of reference.

\section{Primary and secondary objectives}

The primary endpoint of the COLDFIRE-2 trial is efficacy of IRE for CRLM. Secondary endpoints are progressionfree survival (PFS) and overall survival (OS). Other secondary endpoints are safety, technical success, and the accuracy of ${ }^{18} \mathrm{~F}$-FDG PET-CT and 4-phase liver CT in the detection of LTP after IRE.

Primary efficacy rate is defined as the percentage of target tumors successfully eradicated 12 months after the initial IRE procedure, according to the RECIST and PERCIST criteria. Secondary efficacy rate is defined as the percentage of tumors successfully eradicated 12 months after the initial IRE procedure, including tumors that have undergone successful repeat ablation following identification of LTP [26].

Progression-free survival is defined as the time from the first IRE procedure to the time of radiologic disease progression or death of disease. Overall survival is defined as the time from the IRE procedure until death of disease [27]. Both PFS and OS will be determined

Table 2 Scanning parameters of 18 F-FDG PET-CT and 4-phase liver CT

\begin{tabular}{|c|c|c|c|c|c|c|c|}
\hline & Delay (sec) & Contrast & Matrix & Pixel size $\left(\mathrm{mm}^{2}\right)$ & Slice thickness $(\mathrm{mm})$ & Current (mAs/slice) & Energy (keV) \\
\hline Whole body PET & & ${ }^{18} \mathrm{~F} \mathrm{FDG} \mathrm{(ref)}$ & $144 \times 144$ & $4 \times 4$ & 5 & & \\
\hline Low-dose CT & & - & $512 \times 512$ & $1.17 \times 1.17$ & 5 & $30-50$ & 100 \\
\hline 4-phase liver $C T$ & & $100 \mathrm{ml}$ & $512 \times 512$ & $0.68 \times 0.68$ & $4-5$ & & \\
\hline - Precontrast & & Xeneti × 300 & & & & $220 \mathrm{AEC}$ & 120 \\
\hline - Arterial & 12 & & & & & $450 \mathrm{AEC}$ & 80 \\
\hline - Venous & 50 & & & & & $220 \mathrm{AEC}$ & 120 \\
\hline - Hepatic & 220 & & & & & 175 AEC & 120 \\
\hline
\end{tabular}


Table 3 Study form for reviewers' results (per lesion)

\begin{tabular}{|c|c|c|}
\hline \multicolumn{2}{|c|}{ Study number } & \multirow{2}{*}{$\begin{array}{l}\text { Reviewer number } \\
\text { Explanation }\end{array}$} \\
\hline Score & Definition & \\
\hline 1 & Normal & Confident that no tumor recurrence is present in the ablation zone \\
\hline 2 & Probably benign & The appearance of the ablated lesion is compatible with post-ablational inflammation or rim-like characteristics \\
\hline 3 & Equivocal & There is doubt whether the imaging features indicate local tumor progression or inflammation \\
\hline 4 & Probably malignant & Confident about local progression in the ablation zone \\
\hline 5 & Impaired quality & Quality of the images precludes adequate diagnosis \\
\hline \multicolumn{2}{|c|}{ Comments: } & \\
\hline
\end{tabular}

using the Metabolic Imaging And Marker Integration (MIAMI) criteria as proposed by Hosein and colleagues, which integrates anatomic response parameters (4-phase liver $\mathrm{CT}$ ) with two functional parameters: PET activity and CEA levels [28]. For safety analysis, all major adverse events and all adverse events occurring during or within 12 months after IRE treatment will be recorded according to the Common Terminology Criteria of Adverse Events (CTCAE) v4.0 [29]. Pain assessment using the visual analogue scale (VAS) and patient analgesic consumption will be recorded. Patency of vessels and bile ducts on follow-up cross-sectional imaging will be analyzed to identify late complications. Technical success is defined as (1) the ability to successfully deliver all planned pulses according to protocol and (2) complete lesion coverage on post-procedure ceCT or IOUS, and 24-48 h post-procedure MRI [26].

\section{Sample size calculation and statistical considerations}

Based on the current literature our hypothesis is that $10 \%$ of the treated tumors will recur after the initial treatment (primary technique efficacy $90 \%$ ), which implies a local recurrence rate of $0.1[5,30]$. Choosing a target width of 0.25 with $\mathrm{p} \leq 0.05$, we used the confidence interval formula "Exact" for two-sided confidence intervals for one proportion (Clopper-Pearson) [31, 32]. With this formula, actual width is 0.249 , with a $95 \%$ confidence interval of $0.020-0.269$. This calculation results in a sample size of 29 patients. Considering a $5 \%$ loss to follow-up, the total number of patients needed is 31 .

All clinicopathological and procedural variables will be described and analyzed. Continuous variables will be summarized with standard descriptive statistics including means, standard deviations, medians and ranges. Categorical variables will be summarized with frequencies. $P$-values $\leq 0.05$ will be considered statistically significant. Univariate survival analysis will be performed using the Kaplan-Meier method. Differences in local recurrence rate between subgroups like tumor size and adjuvant chemotherapy will be analyzed descriptively since the expected number of recurrences is too small for statistical analysis.
The accuracy of ${ }^{18}$ F-FDG PET-CT and 4-phase liver $\mathrm{CT}$ in diagnosing local recurrences will be investigated by comparison to the reference standard. Interobservervariability will be determined using Cohen's Kappa. Sensitivity and specificity of both techniques will be calculated with their respective $95 \%$ confidence intervals. McNemar's test is used to assess a statistically significant difference between both imaging modalities.

\section{Discussion}

\section{IRE for colorectal liver metastases}

New cancer treatments are typically best defined from phase III randomized trials comparing the investigated therapy with the current standard. However, in the field of local tumor ablation, this has proven a difficult challenge. Since its introduction decades ago, the number of randomized trials remains limited. An attempt to organize a trial comparing RFA to surgical resection has failed (French FFCD 2002-02) and it is unlikely that another trial can be organized in the near future [33]. The current literature on the clinical application of IRE is scarce with no randomized controlled trials. However, because the indication for IRE lies with tumors in which no other suitable local therapy is available, a randomized trial comparing IRE to e.g. surgical resection or thermal ablation is not unrealistic at this point in time.

Results of single-arm studies on hepatic IRE report a high variation in efficacy, with local recurrence rates between $67-100 \%$ [17]. Similar to RFA, efficacy is higher for tumors $\leq 3 \mathrm{~cm}$. However, since IRE is only used as a 'last resort' curative treatment option in patients that would otherwise receive palliative chemotherapy, these early efficacy rates are promising and encourage larger prospective studies.

\section{Feasibility}

Intraprocedural monitoring and control of ablation play a critical role in the success of tumor ablation [34]. The feasibility of real-time monitoring with US during hepatic IRE has been demonstrated in both animal and human studies. The ablation zone immediately appears as a hypoechoic area with well-defined 
margins $[16,35,36]$. Immediate postprocedure ceCT also shows a well-defined hypodense area on the portal venous phase with variable periablational hyperenhancement $[37,38]$. The size and shape of the IRE ablation zone on both US and CT has proven to correlate reliably with the pathologically defined zone of cell death [36, 37]. These imaging modalities could therefore be used to ensure that the realm of ablation encompasses the originally targeted volume with a sufficient margin [15], which is a focus of the presented trial.

\section{Imaging follow-up}

The main concern following tumor ablation is the risk of developing LTP. Early diagnosis of LTP is imperative because repeated treatment can still effectuate complete tumor clearance, especially for smaller recurrences. $\mathrm{CeCT}$ is the current mainstay of staging and follow-up [39]. One shortcoming of ceCT in the monitoring of post-ablative lesions for recurrent disease is the presence of post-ablation effects. Because reactive tissue and viable tumor can both present as hypodensity around the ablated lesion, their distinction can be difficult [24]. Due to the visualization of increased glucose metabolism in tumor cells, ${ }^{18} \mathrm{~F}$-FDG PET has proven a very sensitive and accurate tool for the diagnosis of tumor manifestations in patients with colorectal carcinoma [39]. Using ${ }^{18} \mathrm{~F}$-FDG PET-CT, ${ }^{18}$ F-FDG PET images are combined with $\mathrm{CT}$ in a single session. Anatomical and morphological information from $\mathrm{CT}$ is used to increase the precision of localization, extent, and characterization of lesions detected by ${ }^{18}$ F-FDG PET [21]. Several studies have shown the superiority of ${ }^{18} \mathrm{~F}$-FDG PET-CT over morphologic imaging alone in the follow-up after ablation of CRLM: sensitivity and specificity for the detection of LTP are $92 \% 100 \%$ for ${ }^{18}$ F-FDG PETCT compared to $83 \%$ and $100 \%$ for ceCT [24]. However, the diagnostic accuracy of ${ }^{18} \mathrm{~F}$-FDG PET and ${ }^{18}$ F-FDG PET-CT is strongly affected by chemotherapy, so for patients receiving chemotherapy during follow-up after IRE ceCT may prove the most reliable imaging modality.

After IRE, immediate ceCT shows a hypodense ablation zone that does not enhance post-contrast. A transient peripheral rim of contrast enhancement can be present, representing hyperemia [15]. In the months after ablation, the ablation zone slowly decreases in size and should not show uptake of contrast. PET scans show a dynamic response to the IRE ablation. Three days following IRE, an ${ }^{18}$ F-FDG-avid peripheral zone surrounding the ablated region appears. This initial increase in uptake at the periphery of the IRE region may be explained by an inflammatory response, increasing metabolic activity at the targeted region as the cellular debris is removed from the ablation site [15]. In our experience the inflammatory response can persist for several months, which renders evaluation of the ablation zone with ${ }^{18} \mathrm{~F}$-FDG PET difficult. On the contrary, ablated lesions that show focal rather than rim-like uptake in the periphery are considered suspect for local recurrence. Because much is still unknown about the imaging characteristics of liver lesions treated with IRE, standardized follow-up regimens are lacking. The COLDFIRE-2 trial focuses on the typical imaging characteristics of electroporated CRLM over time. The gold standard for LTP is histologic confirmation. With biopsies taken from all suspicious lesions prior to re-treatment, the trial also assesses the accuracy of the pre-defined definition of LTP on PET-CT and ceCT.

A potentially curative treatment option for a group of patients that is currently offered chemotherapy with palliative intent is of major importance. IRE may prove a safe and valuable fortification in the armory of interventional oncologists treating patients with liver tumors. The aim of the COLDFIRE-2 trial is to contribute to the available evidence on IRE with respect to safety, efficacy, imaging characteristics and follow-up guidelines. The results of the proposed trial may represent an important step towards the implementation of IRE for central liver tumors in the clinical setting.

\section{Abbreviations \\ ASA: American Society of Anesthesiologists; CEA: Carcinoembryonic antigen ceCT: Contrast-enhanced computed tomography; CRLM: Colorectal liver metastases; CTCAE: Common terminology criteria of adverse events; ${ }^{18}$ F-FDG: ${ }^{18}$ F-fluorodeoxyglucose; IOUS: Intra-operative ultrasound: IRE: Irreversible electroporation; LTP: Local tumor progression; ceMRI: Contrast enhanced MRI; MWA: Microwave ablation; NYHA: New York Heart Association; PET: Positron emission tomography; RFA: Radiofrequency ablation; VAS: Visual analogue scale.}

\section{Competing interests}

The authors declare that they have no competing interests and there is no funding to be reported. The study protocol has not undergone peer review by a funding body.

\section{Authors' contributions}

HS: trial coordinator, trial conception and design, data collection, drafting and revising the manuscript. LV: data collection and trial coordinator, revising the manuscript. KN: trial conception and design, drafting and revising the manuscript. AvT: trial conception and design, revising the manuscript. EC: trial conception and design, reviewer of PET-CT images, revising the manuscript. CVK: practical assistance in trial conception and design, revising the manuscript. BvdM: reviewer of $C T$ images, revising the manuscript. JvdB: trial conception and design, reviewer of ceCT images, revising the manuscript. PvdT: principal investigator, trial conception and design, drafting and revising the manuscript. MM: principal investigator, performing percutaneous IRE procedures, trial conception and design, supervising the drafting and revising of the manuscript. All authors approved of the final version submitted.

\section{Author details}

${ }^{1}$ Department of Radiology and Nuclear Medicine, VU University Medical Center, de Boelelaan 1117, 1081 HV Amsterdam, The Netherlands.

Department of Surgery, VU University Medical Center, de Boelelaan 1117, 1081 HV Amsterdam, The Netherlands. 


\section{References}

1. Rempp H, Boss A, Helmberger T, Pereira P. The current role of minimally invasive therapies in the management of liver tumors. Abdom Imaging. 2011;36:635-47.

2. Abdalla EK, Vauthey J-N, Ellis LM, Ellis V, Pollock R, Broglio KR, et al. Recurrence and outcomes following hepatic resection, radiofrequency ablation, and combined resection/ablation for colorectal liver metastases. Ann Surg Oncol. 2004;239:818-25. discussions 825-7.

3. Gleisner A, Choti MA, Assumpcao L, Nathan H, Schulick R, Pawlik T. Recurrence and survival following hepatic resection, radiofrequency ablation, and combined resection-radiofrequency ablation. Arch Surg. 2014;143:1204-12

4. De Baere T, Elias D, Dromain C, Gamal El Din M, Kuoch V, Ducreux M, et al. Hepatic Metastases with a Mean Follow-Up of More Than 1 Year. AJR Am J Roentgenol. 2000;175(December):1619-25.

5. Cannon R, Ellis S, Hayes D, Narayanan G, Martin RCG. Safety and early efficacy of irreversible electroporation for hepatic tumors in proximity to vital structures. J Surg Oncol. 2012;107(October):544-9.

6. Vogl TJ, Farshid P, Naguib NNN, Darvishi A, Bazrafshan B, Mbalisike E, et al. Thermal ablation of liver metastases from colorectal cancer: radiofrequency, microwave and laser ablation therapies. Radiol Med. 2014;119:451-61.

7. Van Tilborg AAJM, Meijerink MR, Sietses C, Van Waesberghe JHTM, Mackintosh $\mathrm{MO}$, Meijer S, et al. Long-term results of radiofrequency ablation for unresectable colorectal liver metastases: a potentially curative intervention. Br J Radiol. 2011;84:556-65.

8. Lu DSK, Yu NC, Raman SS, Limanond P, Lassman C, Murray K, et al. Radiofrequency Ablation of Hepatocellular Carcinoma: Treatment Success as Defined by Histologic Examination of the Explanted Liver. Radiology. 2005;234:954-60.

9. Van den Bos W, Muller BG, de la Rosette JJCMH. A randomized controlled trial on focal therapy for localized prostate carcinoma: hemiablation versus complete ablation with irreversible electroporation. J Endourol. 2013;27:262-4

10. Davalos RV, Mir LM, Rubinsky B. Tissue Ablation with Irreversible Electroporation. Ann Biomed Eng. 2005;33:223-31.

11. Maor E, Ivorra A, Mitchell JJ, Rubinsky B. Vascular smooth muscle cells ablation with endovascular nonthermal irreversible electroporation. J Vasc Interv Radiol. 2010;21:1708-15.

12. Narayanan G, Arora G, Quintana D, Mukkamalla U, Barbery KJ, Hosein P, et al. Vessel patency post irreversible electroporation (IRE) ablation: a 15 month follow-up. J Vasc Interv Radiol. 2012;23:S53.

13. Silk MT, Wimmer T, Lee KS, Srimathveeravalli G, Brown KT, Kingham PT, et al. Percutaneous Ablation of Peribiliary Tumors with Irreversible Electroporation. J Vasc Interv Radiol. 2014;25:112-8.

14. Faroja M, Ahmed M, Appelbaum L, Ben-David E, Moussa M, Sosna J, et al. Irreversible Electroporation Ablation: Is All the Damage Nonthermal? Radiology. 2012;266:462-70.

15. Neal II RE, Cheung W, Kavnoudias H, Thomson KR. Spectrum of imaging and characteristics for liver tumors treated with irreversible electroporation. J Biomed Sci Engin. 2012;2012:11-5.

16. Scheffer HJ, Nielsen $K$, van Tilborg AA, Vieveen JM, Bouwman RA, Kazemier $\mathrm{G}$, et al. Ablation of colorectal liver metastases by irreversible electroporation: results of the COLDFIRE-I ablate-and-resect study. Eur Radiol. 2014:24:2467-75.

17. Scheffer HJ, Nielsen K, de Jong MC, van Tilborg AAJM, Vieveen JM, Bouwman AR, et al. Irreversible Electroporation for Nonthermal Tumor Ablation in the Clinical Setting: A Systematic Review of Safety and Efficacy. J Vasc Interv Radiol. 2014;25:997-1011.

18. Dutton SJ, Kenealy N, Love SB, Wasan HS, Sharma RA. FOXFIRE protocol: an open-label, randomised, phase III trial of 5-fluorouracil, oxaliplatin and folinic acid $(\mathrm{OxMdG})$ with or without interventional Selective Internal Radiation Therapy (SIRT) as first-line treatment for patients with unresectable liver-on. BMC Cancer. 2014;14:497.

19. Van Tilborg AAJM, Scheffer HJ, Nielsen K, van Waesberghe JHTM, Comans EF, van Kuijk C, et al. Transcatheter CT Arterial Portography and CT Hepatic Arteriography for Liver Tumor Visualization during Percutaneous Ablation. J Vasc Interv Radiol. 2014;25:1101-11.

20. Knavel EM, Brace CL. Tumor ablation: common modalities and general practices. Tech Vasc Interv Radiol. 2013;16:192-200.

21. Boellaard R, O'Doherty MJ, Weber WA, Mottaghy FM, Lonsdale MN, Stroobants SG, et al. FDG PET and PET/CT: EANM procedure guidelines for tumour PET imaging: version 1.0. Eur J Nucl Med Mol Imaging. 2010;37:181-200.
22. Eisenhauer EA, Therasse P, Bogaerts J, Schwartz LH, Sargent D, Ford R, et al New response evaluation criteria in solid tumours: Revised RECIST guideline (version 1.1). Eur J Cancer. 2009;45:228-47.

23. Wahl RL, Jacene H, Kasamon Y, Lodge MA. Response Criteria in Solid Tumors. J Nucl Med. 2009;50 Suppl 1:122-50.

24. Nielsen K, van Tilborg AAJM, Scheffer HJ, Meijerink MR, Klerk ESMDL- D,

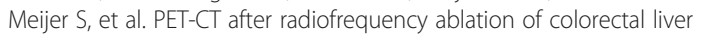
metastases: Suggestions for timing and image interpretation. Eur J Radiol. 2013;82(12):2169-75.

25. Nielsen K, Scheffer HJ, Pieters IC, van Tilborg AAJM, van Waesberghe J-HTM, Oprea-Lager DE, et al. The use of PET-MRI in the follow-up after radiofrequency- and microwave ablation of colorectal liver metastases. BMC Med Imaging. 2014;14:27.

26. Ahmed M. Image-guided Tumor Ablation: Standardization of Terminology and Reporting Criteria - A 10-Year Update. Radiology. 2014;273:241-60.

27. Parmar M, Machin D. Survival Analysis: A Practical Approach. Chichester: John Wiley \& Sons; 1996

28. Hosein PJ, Echenique A, Loaiza-Bonilla A, Froud T, Barbery K, Rocha Lima $\mathrm{CM}$, et al. Percutaneous Irreversible Electroporation for the Treatment of Colorectal Cancer Liver Metastases with a Proposal for a New Response Evaluation System. J Vasc Interv Radiol. 2014;25:1233-9.

29. Common Terminology criteria for Adverse Events version 4.0. U.S. Department of Health and Human Services, National Institutes of Health, National Cancer Institute. [http://evs.nci.nih.gov/ftp1/CTCAE/CTCAE_4.03_ 2010-06-14_QuickReference_5x7.pdf]. Accessed 14 June 2010.

30. Kingham TP, Karkar AM, D'Angelica MI, Allen PJ, Dematteo RP, Getrajdman $\mathrm{Gl}$, et al. Ablation of Perivascular Hepatic Malignant Tumors with Irreversible Electroporation. J Am Coll Surg. 2012;215:379-87.

31. Fleiss $J$, Levin B, Paik MC. Statistical Methods for Rates and Proportions. Thirdth ed. New York: John Wiley \& Sons; 2003.

32. Newcombe RG. Two sided confidence intervals for the single proportion: comparison of seven methods. Stat Med. 1998;17:857-72.

33. Tanis E, Nordlinger B, Mauer M, Sorbye H, van Coevorden F, Gruenberger T, et al. Local recurrence rates after radiofrequency ablation or resection of colorectal liver metastases. Analysis of the European Organisation for Research and Treatment of Cancer \#40004 and \#40983. Eur J Cancer. 2014;50:912-9.

34. Appelbaum L, Mahgerefteh SY, Sosna J, Goldberg SN. Image-guided fusion and navigation: applications in tumor ablation. Tech Vasc Interv Radiol. 2013;16:287-95.

35. Lee EW, Chen C, Prieto VE, Dry SM, Loh CT, Kee ST. Advanced hepatic ablation technique for creating complete cell death: irreversible electroporation. Radiology. 2010;255:426-33.

36. Appelbaum L, Ben-David E, Sosna J, Nissenbaum Y, Goldberg SN. US Findings after Irreversible Electroporation Ablation: radiologic-pathologic correlation. Radiology. 2012;262:117-25.

37. Lee YJ, Lu DSK, Osuagwu F, Lassman C. Irreversible electroporation in porcine liver: acute computed tomography appearance of ablation zone with histopathologic correlation. J Comput Assist Tomogr. 2013:37:154-8.

38. Lee EW, Wong D, Tafti BA, Prieto V, Totonchy M, Hilton J, et al. Irreversible electroporation in eradication of rabbit VX2 liver tumor. J Vasc Interv Radiol. 2012;23:833-40

39. Kuehl H, Antoch $G$, Stergar H, Veit-Haibach P, Rosenbaum-Krumme S, Vogt F, et al. Comparison of FDG-PET, PET/CT and MRI for follow-up of colorectal liver metastases treated with radiofrequency ablation: initial results. Eur J Radiol. 2008;67:362-71.

\section{Submit your next manuscript to BioMed Central and take full advantage of:}

- Convenient online submission

- Thorough peer review

- No space constraints or color figure charges

- Immediate publication on acceptance

- Inclusion in PubMed, CAS, Scopus and Google Scholar

- Research which is freely available for redistribution 